Borneo Journal of Sciences and Technology, Volume (1), Issue (2), Pages: 27-31

DOI: https://doi.org/10.35370/bjost.2019.1.2-04

e-ISSN: 2672-7439

(C) 2018, UCTS Publisher.

Submitted: $22^{\text {nd }}$ March 2019

Accepted: $15^{\text {th }}$ April 2019

Published: $31^{\text {st }}$ July 2019

\title{
Synthesis and Characterization of Magnetite Nanoparticles by Chemical Co-Precipitation
}

\author{
${ }^{1}$ Cheong Kah Yein, ${ }^{1}$ Kong Sieng Huat, ${ }^{2}$ Mohd. Ambar Yarmo \\ ${ }^{1}$ School of Foundation Studies, University College of Technology Sarawak, 96000 Sibu, Sarawak, \\ Malaysia \\ ${ }^{2}$ Universiti Kebangsaan Malaysia, 43600 UKM Bangi, Selangor, Malaysia
}

\begin{abstract}
A modified controlled chemical co-precipitation of alkaline aqueous ferrous and ferric salt solution at $\mathrm{pH}$ 8 with continuous addition of ammonia solution $25 \%$ under a degassed atmosphere was performed to synthesis magnetite $\left(\mathrm{Fe}_{3} \mathrm{O}_{4}\right)$ nanoparticles. Formation of magnetite nanoparticles was conducted by adjusting the ferric to ferrous ions in the ratio of 1:1, 1:2 and 2:1. Further investigation on the surfactant-coated magnetite nanoparticles by using $8 \%$ surfactant sodium dodecyl sulphate (SDS) was also studied. The synthesized magnetite nanoparticles were characterized by Transmission electron microscope (TEM), Fourier transform infrared spectroscopy (FTIR), X-ray photoelectron spectroscopy (XPS) and X-ray diffractometer (XRD). TEM results shows that magnetite nanoparticles which were synthesized with ferric/ferrous ratio 2:1 are in sphere shape and have the smallest particle size distribution range which is about $12-17 \mathrm{~nm}$. The particles size distribution range of coated magnetite was decreased to 11-15 nm after coated with $8 \%$ surfactant SDS. XPS results indicated that the produced magnetite nanoparticles consisted of elemental iron and oxygen at $72.76 \%$ and $22.27 \%$ respectively. The phase and face-centered cubic structure of magnetite nanoparticle was also confirmed by XRD. Magnetite nanoparticle synthesized with ferric to ferrous ratio of 2:1 and coated with $8 \%$ surfactant SDS shows the best crystallinity among all samples with particle distribution size range from $11-15 \mathrm{~nm}$.
\end{abstract}

Keywords: Magnetite nanoparticle, iron oxide, surfactant, co-precipitation, ferrous, ferric

\section{INTRODUCTION}

To date, magnetite $\left(\mathrm{Fe}_{3} \mathrm{O}_{4}\right)$ nanoparticle is a wellknown material and it has gained attention because of its unique chemical and physical properties. Magnetite is a black luster and magnetic compound that containing both ferrous and ferric iron species that are arranged in an inverse spinel crystal structure [1]. Magnetite exhibits properties such as superparamagnetism, large surface area, good biocompatibility and low-toxicity [2]. These interesting properties have been intensively studied and lead to its usage in various industrial applications such as pigment synthesis for paints, bank cheque and porcelain [3], catalysis [4], biosensor [5] as well as in biomedical application [6].

Currently, there are several chemical methods available for synthesizing magnetite nanoparticle such as sol-gel technique, co-precipitation, non-hydrolytic method, thermal decomposition and micro emulsion method [7]. Typically, aqueous co-precipitation of ferrous ion and ferric ion with addition of ammonia is commonly practiced to prepare magnetite nanoparticle due to its simple, environment-friendly and economical process which only use cheap deionized water and iron with no harmful byproducts formed throughout the synthesis reaction [7]. Besides, the rapid preparation steps with none using of organic solvent is also one of the advantages to adopt this synthesis method rather than others synthesis approaches which mentioned earlier. However, the control of $\mathrm{pH}$ in precipitation reaction is very important in controlling the size of nanoparticle [2].

Furthermore, due to the tendency of agglomeration in magnetite nanoparticles which limit its application in medical diagnostic due to its chemically-instability properties, several stabilizers such as polyethylene glycol, polyvinyl alcohol and galactose-carrying polymer were studied to stabilize magnetite nanoparticles [8]. Thus, a controlled synthetic method of magnetite nanoparticle need to be optimized for advanced application. Herein, the main objective of this study focused on the effect of different ferric to ferrous ions ratio $(1: 1,1: 2$ and $2: 1)$ on the formation of magnetite nanoparticles. The effect of ferric/ferrous ratio on the surface morphology and nanoparticles size

Corresponding Author: Cheong Kah Yein, Affliation: University College of Technology Sarawak, (+6) 084-367519, email:cheong.kah.yein@ucts.edu.my 
were discussed in this study. Further investigation on the addition of anionic surfactant $\mathrm{SDS}\left(\mathrm{NaC}_{12} \mathrm{H}_{25} \mathrm{SO}_{4}\right)$ to the synthesized magnetite nanoparticle was also conducted in this study. Overall, its application performance can be enhanced at nanometer level when synthesis method provide uniform and well defined particle.

\section{MATERIALS AND METHODS}

\section{A. SYNTHESIS OF MAGNETITE NANOPARTICLE}

In this experiment, the formation of magnetite nanoparticle was conducted by adjusting the ferric to ferrous ions ratio from $1: 1,1: 2$ and $2: 1$. The amount of surfactant SDS used for coating of magnetite nanoparticle was $8 \%$. Iron (II) sulphate heptahydrate $\left(\mathrm{FeSO}_{4} .7 \mathrm{H}_{2} \mathrm{O}\right)$, iron (III) chloride $\left(\mathrm{FeCl}_{3}\right)$ and ammonia solution $25 \%$ were used as the reagents. In this experiment, $\mathrm{FeCl}_{3}$ and $\mathrm{FeSO}_{4} \cdot 7 \mathrm{H}_{2} \mathrm{O}$ were mixed and stirred in $60 \mathrm{~mL}$ deionized water. Further investigation on the SDS surfactant-coated magnetite nanoparticle was studied by adding surfactant SDS into the salt mixture, then followed by drop-wise addition of $10 \mathrm{~mL}$ mixture of deionized water: ammonia solution $25 \%$ (1:1). The salt mixture was stirred continuously for an hour and the black precipitate generated gradually. The resultant black precipitates were washed several times with deionized water until the $\mathrm{pH}$ reached 8 . The black precipitate was then washed with acetone solution and dried for further characterizations.

\section{B. CHARACTERIZATION}

The surface morphology and size of magnetite nanoparticles were characterized using the transmission electron microscope (TEM, Philips CM12). The optimum nanoparticle synthesized at ratio $2: 1$ were then further characterized using Fourier transform infrared spectroscopy (FTIR, Perkin Elmer) to identify the presence of iron oxide and SDS surfactant in the sample between 400-4000 $\mathrm{cm}^{-1}$. Besides, its elemental composition and chemical state of magnetite nanoparticle were also characterized using X-ray photoelectron spectroscopy (XPS, Kratos Axis Ultra DLD) at wide and narrow scanning resolution. X-ray diffraction (XRD) data of the sample was also collected with diffractometer (Siemen D-5000) with $\mathrm{CuK} \alpha 2 \theta$ radiation source in the range of $25-70^{\circ}$.

\section{RESULTS AND DISCUSSION}

Black color precipitates were formed due to the precipitation of magnetite nanoparticles from ferric and ferrous salts mixture [9]. The formation of magnetite nanoparticles likely occurred by the reaction between ferrous hydroxide, $\mathrm{Fe}(\mathrm{OH})_{2}$ and ferric oxyhydroxide, $\mathrm{FeO}(\mathrm{OH})$ formed when ammonia solution was added into the ferrous/ferric salt mixture [10]. By adjusting the ferric and ferrous ions ratio, the size and shape of magnetite nanoparticles were analyzed and compared using electron microscope analysis. Prior to the electron microscope analysis, magnetite nanoparticle was dispersed using ultrasonicator. The TEM images of the synthesized samples are shown in Figure 1 to Figure 4. It could be seen from the TEM images that all the synthesized magnetite nanoparticles were roughly spherical in shapes [11]. It was observed that the particle size distribution range between 12-17 nm was recorded for sample synthesized at a ratio of 2:1 (Figure 1).

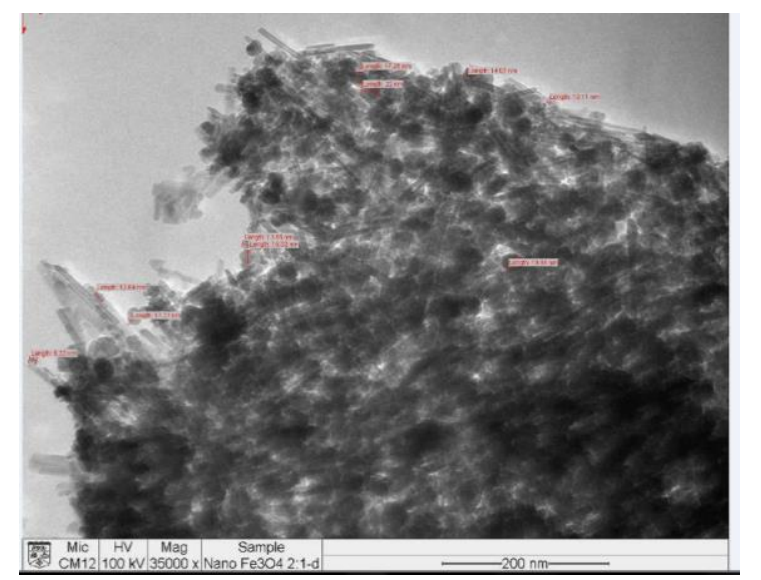

Figure 1: Transmission Electron Micrograph of Magnetite Nanoparticle Formed in Ferric: Ferrous Ratio 2:1

Whereas, sample synthesized at $1: 1$ ratio (Figure 2) and 1:2 ratio (Figure 3) were found to have particle size distribution range from 11-23 nm and 30$50 \mathrm{~nm}$ respectively. Besides, it was also observed from TEM images that magnetite nanoparticles were mainly agglomerated as compared to magnetite nanoparticles that were coated with SDS surfactant (Figure 4). Magnetite nanoparticles that were surfactant-coated revealed well-dispersed and exhibits the best crystallized particles size of 11-15 nm. Similar effect was also observed in [12] which shows that addition of $2 \%$ SDS was able to form the smallest magnetite particle size with $8 \mathrm{~nm}$. This could be due the reason that the presence of SDS in magnetite nanoparticle which acts as a capping agent is able to limit or control the size of magnetite nuclei from agglomeration during the co-precipitation process [12]. 


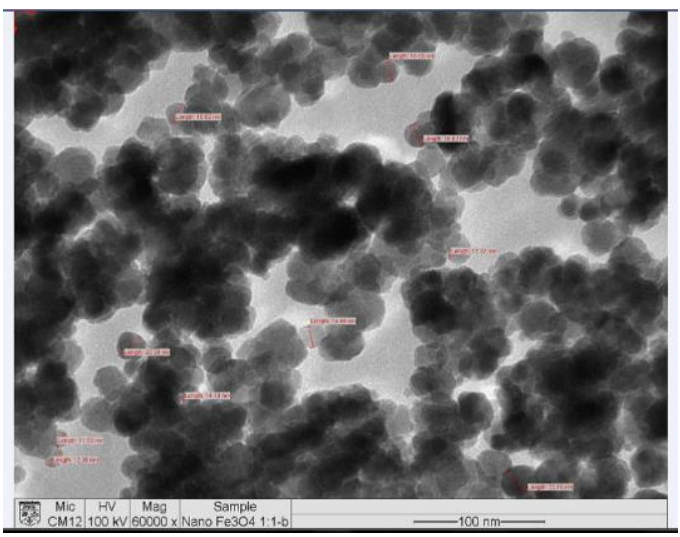

Figure 2: Transmission Electron Micrograph of Magnetite Nanoparticle Formed in Ferric: Ferrous Ratio $1: 1$

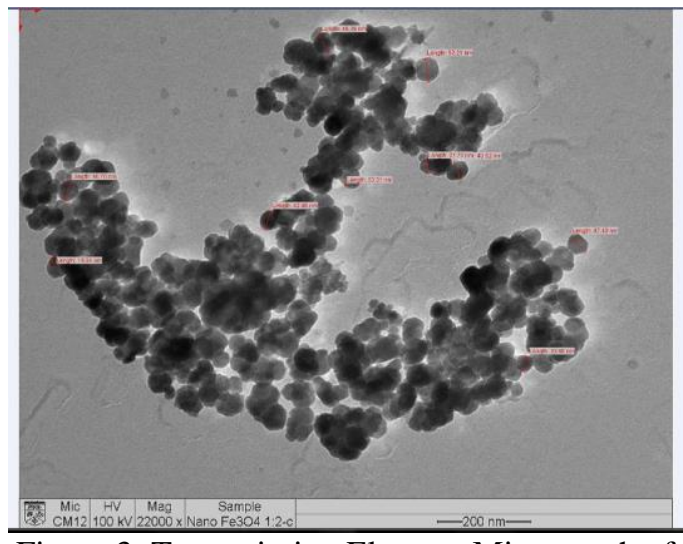

Figure 3: Transmission Electron Micrograph of Magnetite Nanoparticle Formed in Ferric: Ferrous Ratio $1: 2$

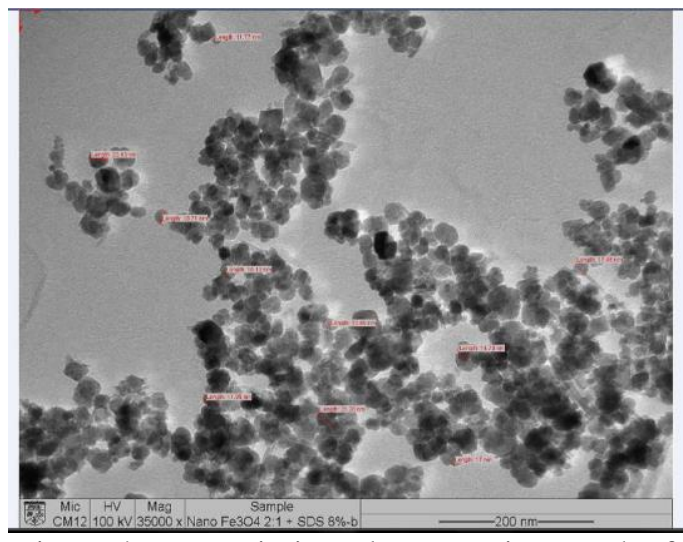

Figure 4: Transmission Electron Micrograph of

Magnetite Nanoparticle Formed in Ferric: Ferrous Ratio 2:1 with Sodium Dodecyl Sulphate (SDS) Coating

The FTIR results (Figure 5a \& Figure 5b) indicated that the existence of stretching peaks at 579 and $630 \mathrm{~cm}^{-1}$ [6] in both samples synthesized at ratio 2:1 and sample coated with SDS surfactant are corresponded to the $\mathrm{Fe}-\mathrm{O}$ bond in magnetite nanoparticles. Meanwhile $\mathrm{OH}$ bending identified (Figure 5a \& Figure 5b) at around $1630 \mathrm{~cm}^{-1}$ [6] indicating the existence of iron hydroxide as well. FTIR analysis was also performed to confirm the coating on magnetite nanoparticle. The band at $1124 \mathrm{~cm}^{-1}$ as shown in FTIR spectrum (Figure 5b) is attributed to the symmetry $\mathrm{S}=\mathrm{O}$ stretching peak of SDS surfactant. In addition, the $\mathrm{S}-\mathrm{O}$ stretching peaks are also found at $887-1052 \mathrm{~cm}^{-1}$ (Figure 5b) [13]. This is an indication of the SDS surfactant was successfully coated on the superparamagnetic magnetite nanoparticle.

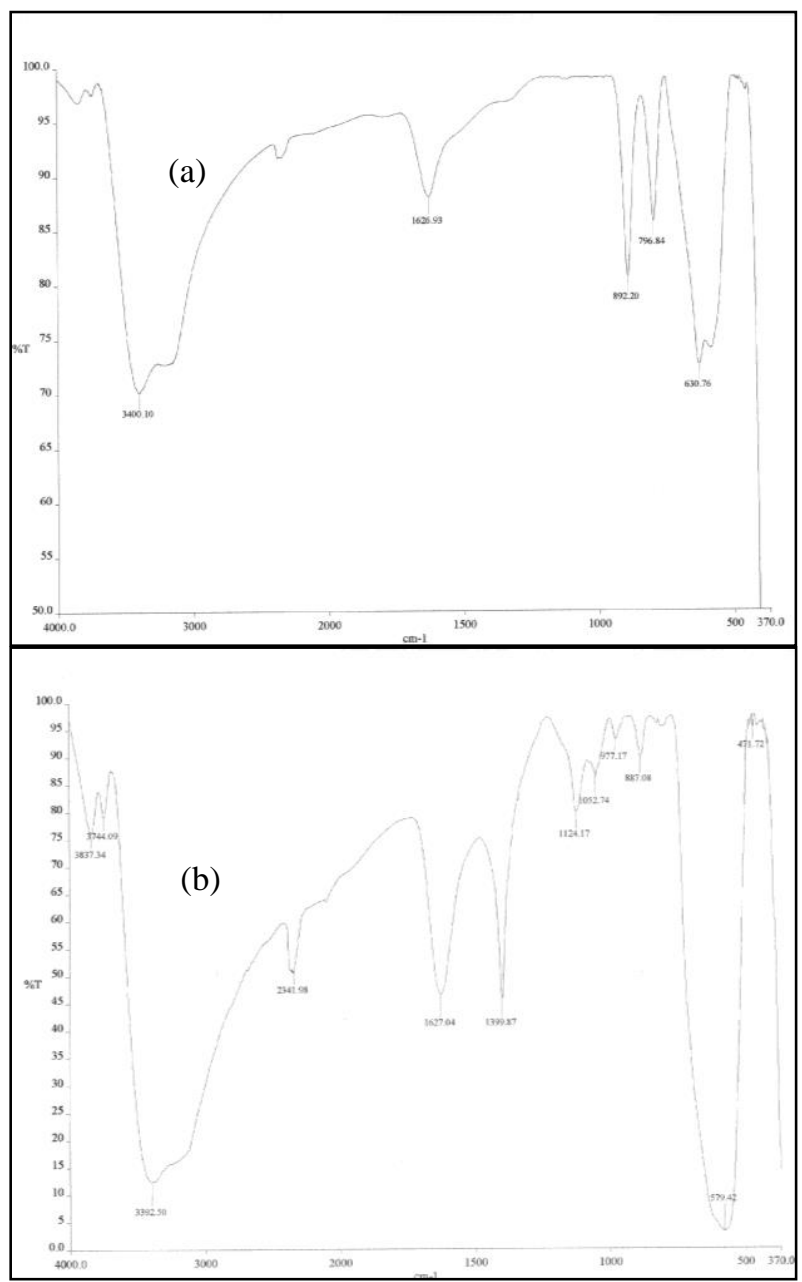

Figure 5: FTIR Spectroscopy Spectra of Magnetite Synthesized at Ratio 2:1

(a) Without Coating (b) Coated with Sodium Dodecyl Sulphate (SDS) 
Elemental composition and chemical properties of synthesized magnetite nanoparticle at ratio 2:1 were also analyzed using $\mathrm{X}$-ray photoelectron spectroscopy. Spectrum (Figure 6a) resulted from XPS indicated that the produced magnetite nanoparticles consisted of elemental iron and oxygen at $72.76 \%$ and $22.27 \%$ respectively. Besides, element iron embedded in magnetite nanoparticles were found exist in two different electronic configuration which is $\mathrm{Fe} 2 \mathrm{p}_{1 / 2}$ and $\mathrm{Fe} 2 \mathrm{p}_{3 / 2}$ based on the spectrum (Figure 6b) resulted from XPS [14]. Both peaks found in $\mathrm{Fe} 2 \mathrm{p}_{3 / 2}$ and $\mathrm{Fe} 2 \mathrm{p}_{1 / 2}$ were found consisted of two overlapping peaks at binding energy 709-711 eV and 723-724 eV respectively. These binding energies analyzed from the XPS spectrum are same with reference value for magnetite nanoparticle [14]. This further confirmed that the nanoparticle synthesized is consisted of magnetite.

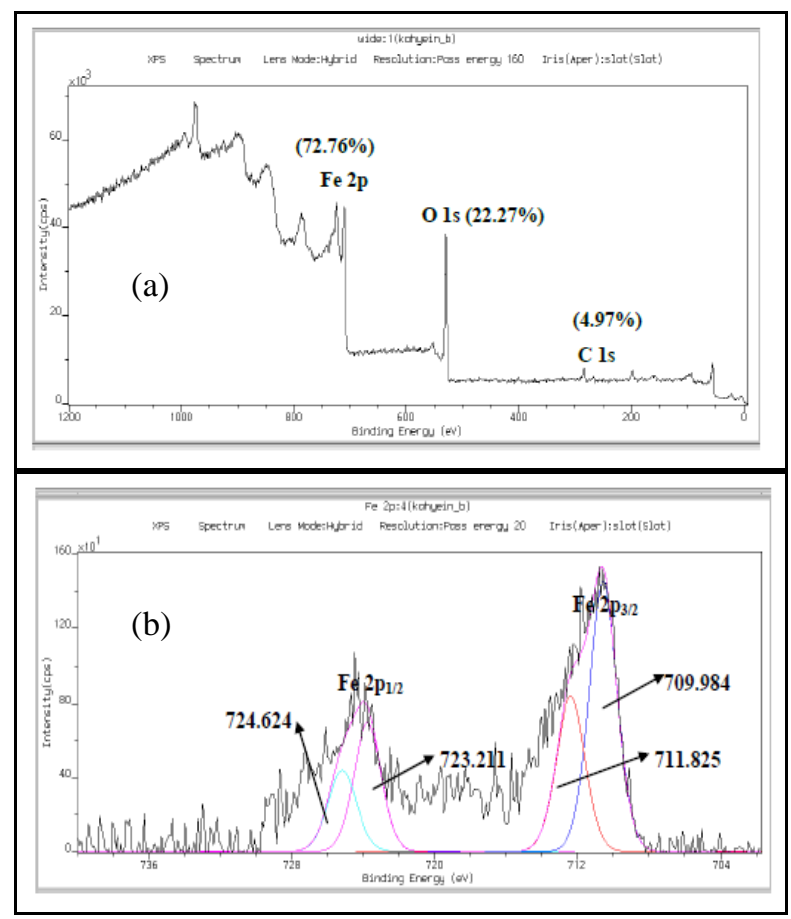

Figure 6: X-Ray Photoelectron Spectroscopy Spectrum of Magnetite Nanoparticle Synthesized at Ratio 2:1

XRD measurement was carried out to identify the phase structure of the magnetite nanoparticles. The reflection peak position and intensities relatively matched the magnetite nanoparticle [15] as shown in Figure 7. All samples demonstrated theoretical face-centered cubic structure with six diffraction peaks corresponding to (2 2 0), (3 11 1), (4 00 0), (4 2 2), (5 11 1), (4 4 l 0 ) [15]. These results showed that magnetite nanoparticle synthesized were consisted of face-centered cubic structure [16] and do not contain other types of iron oxide e.g. $\alpha-\mathrm{Fe}_{2} \mathrm{O}_{3}$ and $\gamma-\mathrm{Fe}_{2} \mathrm{O}_{3}$ compound based on the diffraction peaks in the XRD spectra [17]. Besides, the coated magnetite nanoparticles showed higher crystallinity than the non-coated magnetite which the latter showed lower intense reflection peaks.

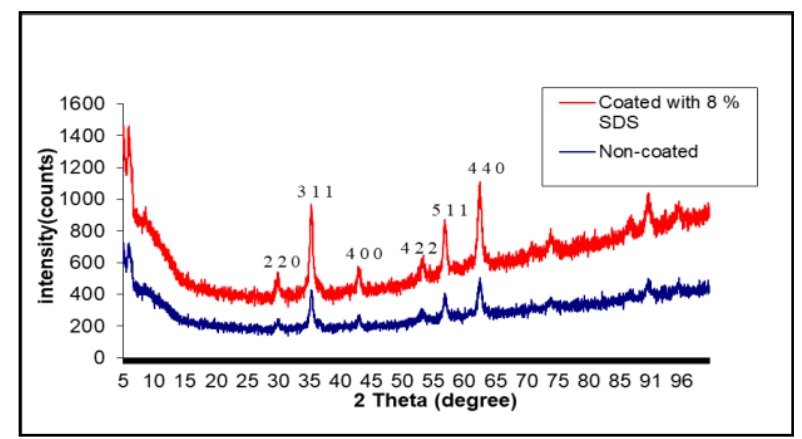

Figure 7: X-Ray Diffraction Pattern of Coated and Non-Coated Magnetite Synthesized at Ratio 2:1

\section{CONCLUSION}

Results indicated that magnetite nanoparticles synthesized at ferric to ferrous ratio 2:1 was found to have the smallest particle size distribution range which is $12-17 \mathrm{~nm}$. It was observed that the addition of SDS surfactant into magnetite nanoparticles would result in the decrease of particle size distribution range and a well-dispersed particle structure. Magnetite nanoparticles synthesized were also confirmed by FTIR spectroscopy analysis and XRD pattern. It can be concluded that the magnetite nanoparticle synthesized with ferric to ferrous ratio of $2: 1$ and coated with $8 \%$ surfactant sodium dodecyl sulphate shows the best crystallinity among all samples with particle distribution size range from 11$15 \mathrm{~nm}$.

\section{ACKNOWLEDGMENT}

The authors wish to thank Universiti Kebangsaan Malaysia (UKM) for the permission to conduct this research and also University College Technology Sarawak (UCTS) for the financial support.

\section{REFERENCES}

[1] Taufiq, A., Triwikantoro, Pratapa, S., and Darminto, D. 2008. Sintesis Partikel Nano $\mathrm{Fe}_{3-}$ ${ }_{x} \mathrm{Mn}_{\mathrm{x}} \mathrm{O}_{4}$ Berbasis Pasir Besi dan Karakterisasi Strucktur serta Kemagnetannya. Jurnal Nanosains \& Nanoteknologi, 1(2), 67-73.

[2] Jiang, C.M., and Lin, X.Q. 2009. Electrochemical Synthesis of $\mathrm{Fe}_{3} \mathrm{O}_{4}-\mathrm{PB}$ Nanoparticles with Core-Shell Structure and Its 
Electrocatalytic Reduction towards $\mathrm{H}_{2} \mathrm{O}_{2}$. Journal of Solid State Electrochemistry, 13(8), 1273-1278.

[3] Teja, A.S., and Koh, P.Y. 2009. Synthesis, Properties and Applications of Magnetic Iron Oxide Nanoparticles. Progress in Crystal Growth and Characterization of Materials, 55, 22-45.

[4] Amara, D., Felner, I., Nowik, I., and Margel, S. 2009. Synthesis and Characterization of $\mathrm{Fe}$ and $\mathrm{Fe}_{3} \mathrm{O}_{4}$ by Thermal Decomposition of Triiron Dodecacarbonyl. Colloids and Surfaces A: Physicochemical and Engineering Aspects, 339(1-3), 106-110.

[5] Loh, K.S., Lee, Y.H., Musa, A., Salmah, A.A., and Zamri, I. 2008. Use of $\mathrm{Fe}_{3} \mathrm{O}_{4}$ Nanoparticles for Enhancement of Biosensor Response to the Herbicide 2,4-dichlorophenoxyacetic Acid, Sensor, 8(9), 5775-5791.

[6] Predoi, D. 2007. A Study on Iron Oxide Nanoparticles Coated with Dextrin Obtained by Coprecipitation. Digest Journal of Nanomaterials and Biostructures, 2(1), 169-173.

[7] Yao, K., Peng, Z., and Fan, X.L. 2009. Preparation of Nanoparticles with an Environment-friendly Approach. Journal of Environmental Sciences, 21(6), 727-730.

[8] Yoo, M.K., Kim, I.Y., Kim, E.M., Jeong, H.J., Lee, C.M., Jeong, Y.Y., Toshihiro Akaike and Cho, S.C. 2007. Superparamagnetic Iron Oxide Nanoparticles Coated with Galactose-Carrying Polymer for Hepatocyte Targeting. Journal of Biomedicine and Biotechnology, (10), 1-9.

[9] Kim, D.K., Zhang, Y., Voi, W., Rao, K.V., and Muhammed, M. 2001. Synthesis and Characterization of Surfactant-Coated Superparamagnetic Monodispersed Iron Oxide Nanoparticles. Journal of Magnetism and Magnetic Material, 225(1-2), 30-36.

[10] Januarita, A.W., Febriana, R., Fajaroh, F., and Setyawan, H. 2010. Sintesa nanopartikel magnetite dengan methode electrokimia. Seminar Rekayasa Kimia dan Proses. Universitas Diponegoro Semarang, August, 4-5, pp: 1-7.

[11] Sun, J., Zhou, S.B., Hou, P., Yang, Y., Weng, J., Li, X.H., and Li, M.Y. 2006. Synthesis and Characterization of Biocompatible $\mathrm{Fe}_{3} \mathrm{O}_{4}$ Nanoparticles. Journal of Biomedical Materials Research Part A, 80A(2), 333-341.

[12] El-kharrag, R., Amin, A., and Greish, Y.E. 2011. Synthesis and Characterization of Mesoporous Sodium Dodecyl Sulfate-Coated Magnetite Nanoparticles. Journal of Ceramic Science and Technology, 02(04), 203-210.

[13] Pavia, D.L., Lampman, G.M., and Kriz, G.S. 2001. Introduction to Spectroscopy. Thomson Learning, Australia.
[14] Moulder, J.H., Stickle, W.F., Sobol, P.E., and Bomben, K.D. 1995. Handbook of X-ray Photoelectron Spectroscopy. Perkin Elmer Corporation, United States of America.

[15] Jing, S., Zhou, S.B., Hou, P., Yang, Y., Weng, J., Li, X.H. \& Li, M.Y. 2006. Synthesis and characterization of Biocompatible $\mathrm{Fe}_{3} \mathrm{O}_{4}$ nanoparticles. Wiley Interscience, 80(2), 333341.

[16] Zhang, X., Zhou, R., Rao, W., Cheng, Y. \& Ekoko, B.G. 2006. Influence of Precipitator Agents $\mathrm{NaOH}$ and $\mathrm{NH}_{4} \mathrm{OH}$ on the Precipitation of $\mathrm{Fe}_{3} \mathrm{O}_{4}$ Nanoparticles Synthesized by Electron Beam Irradiation. Journal of Radioanalytical and Nuclear Chemistry, 270, 285-289.

[17] Jing, X., Yang, H.B., Fu, W.Y., Du, K., Sui, Y.M., Chen, J.J., Zeng, Y., Li, M.H., and Zou, G.T. 2007. Preparation and Magnetic Properties of Magnetic Nanoparticles by Sol-Gel Method. Journal of Magnestism and Magnetics Materials, 309(2), 307-311. 